\title{
Laboreal
}

Volume $4 \mathrm{~N}^{\circ} 2$ | 2008

A inovação

\section{Medir o impacto do trabalho sobre a saúde : estudos longitudinais, sim, mas quais?}

Medir el impacto del trabajo sobre la salud : estudios longitudinales, si, pero cuáles?

Mesurer l'impact du travail sur la santé : du longitudinal, oui, mais lequel?

The measurement of the impact of work on health : we need longitudinal studies, but which ones?

\section{Thomas Coutrot e Loup Wolff}

Tradutor: Cláudia Monteiro, Rita Gil Mata e Marianne Lacomblez

\section{(2) OpenEdition}

\section{Journals}

\section{Edição electrónica}

URL: http://journals.openedition.org/laboreal/11496

ISSN: 1646-5237

\section{Editora}

Universidade do Porto

Refêrencia eletrónica

Thomas Coutrot e Loup Wolff, « Medir o impacto do trabalho sobre a saúde : estudos longitudinais, sim, mas quais? », Laboreal [Online], Volume $4 \mathrm{~N}^{\circ} 2 \mid$ | 2008, posto online no dia 01 dezembro 2008, consultado o 10 outubro 2019. URL : http://journals.openedition.org/laboreal/11496

Este documento foi criado de forma automática no dia 10 outubro 2019.

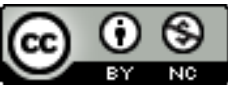

Laboreal está licenciado com uma Licença Creative Commons - Atribuição-NãoComercial 4.0 Internacional. 


\section{Medir o impacto do trabalho sobre a saúde : estudos longitudinais, sim, mas quais?}

Medir el impacto del trabajo sobre la salud : estudios longitudinales, si, pero cuáles?

Mesurer l'impact du travail sur la santé : du longitudinal, oui, mais lequel?

The measurement of the impact of work on health : we need longitudinal studies, but which ones?

\section{Thomas Coutrot e Loup Wolff}

Tradução : Cláudia Monteiro, Rita Gil Mata e Marianne Lacomblez

\section{REFERÊNCIA}

Artigo original : Coutrot, T. \& Wolff, L. (2008). Mesurer l'impact du travail sur la santé : du longitudinal, oui, mais lequel ? PISTES, 10, 2.

1 Para poder avaliar as relações entre exposições profissionais e saúde dos assalariados, os epidemiologistas têm necessidade de dispor de um instrumento de observação adaptado: um inquérito longitudinal de preferência ou, na falta deste, em corte instantâneo (cross-section) com um questionamento retrospectivo sobre as exposições aos factores de risco (Goldberg, 2003). Nos dois casos, é preciso ter informações sobre o modo de vida dos assalariados (factores de risco pessoais) e sobre o seu historial médico, que podem constituir "factores de confusão" susceptíveis de introduzir enviesamentos na medida das relações entre exposições profissionais e saúde, se não forem controlados.

Mas que podemos fazer se os dados de que dispomos não preenchem todas estas condições? De que amplitude são os enviesamentos produzidos pela utilização de um inquérito em corte instantâneo, no qual não conhecemos nem as exposições passadas, 
nem os factores de risco pessoais? E se os dados preenchem todas as condições necessárias, e compreendem a dimensão longitudinal, como especificar o modelo : é necessário explicar a evolução da saúde pelas condições de trabalho à data inicial, ou antes pela evolução das condições de trabalho entre as duas datas de observação?

\section{Metodologia}

30 artigo propõe-se comparar as performances respectivas de uma série de modelos estatísticos ligando as características de trabalho e a saúde, num corte transversal e longitudinal, sobre a mesma base de dados: o inquérito ESTEV "Trabalho, Envelhecimento, Saúde" (ver quadro no final), realizado em 1990 e 1995, com aproximadamente 20000 assalariados, por perto de 1000 médicos de trabalho. Cada uma das famílias de modelos foi aplicada a 29 variáveis, descrevendo o estado de saúde, das quais procura-se explicar a prevalência ou incidência : 16 patologias diagnosticadas pelos médicos, 12 (infra) patologias evocadas pelos assalariados e a má saúde autoavaliada pelos assalariados $\left.{ }^{[}\right]$.

Os modelos incluem todas as variáveis socioprofissionais de controlo (idade, sexo, profissão e categoria socioprofissional, sector de actividade) ${ }^{2}$.

Os dados utilizados : o inquérito ESTEV

O inquérito ESTEV foi iniciado em 1990 por 400 médicos voluntários por ocasião das visitas anuais de medicina do trabalho (Derrienic, Touranchet \& Volkoff, 1996). A amostra inicial era constituída por 21378 assalariados dos dois sexos e de quatro anos de nascimento (em 1938, 1943, 1948 e 1953), tirados por sorteio para o inquérito, vindos de sete regiões. Em 1995, um segundo questionamento abarcou 18695 assalariados, mais de 87 \% da população inicial, revistos por perto de 1000 médicos.

A metodologia original do ESTEV relativamente a outros inquéritos sobre as condições de trabalho ou a saúde no trabalho (Bué, Coutrot \& Puech, 2005) constituiu uma base de dados de uma grande riqueza, não só no campo da saúde (graças à visita médica), mas também no do trabalho (por um auto-questionário), permitindo um controle rigoroso dos efeitos da variável idade, e dotado de uma dimensão longitudinal.

O questionário compreende informações sobre as exposições ${ }^{3}$ actuais e passadas (com as durações de exposição) aos principais riscos (horários atípicos, riscos físicos, químicos e organizacionais) ; sobre as características pessoais (vida familiar, actividade fora do trabalho como desporto ou bricolage, e factores de risco pessoais como o consumo de álcool e tabaco) ; sobre a saúde ("infrapatologias" assinaladas pelos assalariados e patologias diagnosticadas pelos médicos).

O campo de estudo é o conjunto dos indivíduos interrogados em 1990 e em 1995, assalariados em 1990 e em 1995, ou seja 16950 indivíduos (um pouco mais de $79 \%$ da população inicial). Este nuance implica uma redução do tamanho da amostra e sobretudo uma deformação da sua estrutura. Ressalvámos que os indivíduos removidos do estudo, apresentavam em 1990 condições de trabalho mais difíceis e 
uma saúde menos boa que os outros. Este enviesamento de selecção pode afectar a análise do impacto das condições de trabalho sobre a saúde ; com efeito, os indivíduos no início mais fragilizados (e que são, então, a priori os mais susceptíveis de reagir negativamente a uma degradação das suas condições de trabalho), tinham menos hipótese de serem estudados. Mas este viés vai no sentido de uma subestimação dos efeitos : poderemos então considerar os resultados aqui apresentados como avaliações conservadoras das relações entre trabalho e saúde. Para mais detalhes metodológicos, ver (Coutrot \& Wolff, 2005).

5 As cinco famílias de modelos das quais vão ser comparadas as performances são as seguintes :

- Os modelos estáticos simples (ou naifs) explicam a prevalência de uma dada patologia ou infra-patologia em 1995 unicamente em função das exposições de 1995 e das variáveis socioprofissionais características dos assalariados.

- Os modelos estáticos completos acrescentam às variáveis explicativas precedentes as que descrevem o conjunto de características pessoais (situação familiar) e comportamentos potencialmente de risco (tabaco, álcool, desporto, bricolage) disponíveis no ESTEV, assim como o conjunto das patologias anteriores e actuais diagnosticadas pelo médico no momento do inquérito (com a excepção, claro, da patologia a explicar).

- Os modelos estáticos retrospectivos consideram a informação fornecida em 1995 sobre as exposições actuais ou passadas e sobre a sua duração.

- Os modelos longitudinais em nível, para os quais mobilizar-se-á também os dados de 1990, explicam a incidência de uma patologia entre 1990 e 1995 pelo nível de exposições em 1990. Este é o tipo de modelo que é mais classicamente utilizado em epidemiologia. Pode também ser qualificado de modelo "standard" (cf., por exemplo, Derriennic, Monfort, Cassou \& Touranchet, 2001).

- Os modelos longitudinais em variações modelizam a incidência de uma patologia entre 1990 e 1995 em função das características pessoais (em 1995) e da variação das exposições entre 1990 e 1995. Este tipo de modelo é mais raramente utilizado (ver todavia para inquérito STED : Ministério de emprego e da solidariedade, 2001 ; Bertin \& Derriennic, 2001 ; Vézina, Derriennic \& Monfort, 2001).

6 A ambição deste artigo é puramente estatística : não procuramos analisar a pertinência dos modelos de um ponto de vista epidemiológico (ou seja, de julgar a verosimilhança médica das relações estatísticas analisadas), mas comparar as suas performances com a ajuda de critérios estatísticos. A ideia geral que baseia a nossa démarche é a seguinte : um modelo tem um desempenho tanto mais performante quanto permite evidenciar um maior número de relações significativas entre a variável explicada (a saúde, ou seja, aqui, as patologias e infra-patologias) e as variáveis explicativas (as exposições profissionais), que a literatura epidemiológica identificou como os efeitos potenciais. $O$ critério de base é, portanto, o número de odds ratios reportando a um nível de significância de 0,05 em cada família de modelos. Procederemos a cinco comparações entre duas famílias de modelos: compararemos primeiro os modelos naïfs com os modelos estáticos completos, em seguida com os modelos retrospectivos. Compararemos depois os modelos longitudinais em variações com os modelos naïfs, antes de tentar uma comparação global e simultânea do conjunto de famílias de modelos. Para cada uma destas comparações, o critério de base (número de odds ratios significativos) necessitará, para se tornar operacional, da adopção de uma "convenção 
de comparabilidade", que dependerá em cada vez da respectiva estrutura das famílias de modelos comparadas.

\section{Comparação entre os modelos estáticos}

7 A comparação sistemática das estimativas fornecidas sucessivamente pelos três tipos de modelos estáticos propostos (modelos naïf, estático completo e estático retrospectivo) revela a fragilidade do enviesamento induzido pela omissão dos factores ligados à vida pessoal e aos antecedentes médicos.

\subsection{A abordagem naïve faz aparecer numerosos efeitos significativos}

Os modelos estáticos naïfs incluem 3 a 21 relações significativas entre exposições e saúde, com uma média de 9 odds ratios significativos por modelo. Entre as patologias diagnosticadas, as do sistema osteo-articular e muscular, incluindo as perturbações musculo-esqueléticas (frequência de $27 \%$ ), obtêm o número mais elevado de odds ratios significativos (12) para as variáveis descrevendo as exposições profissionais actuais; as perturbações mentais (frequência de $6 \%$ ) incluem 11 odds ratios significativos.

Por outro lado, patologias relativamente mais raras - tais como doenças da pele ( $4.1 \%$ dos assalariados), os cancros (1.4\%) ou as doenças do sistema nervoso (3.2 \%) - não contam cada uma mais que 3 exposições dotadas de odds ratios significativos.

10 As infra-patologias declaradas têm uma relação muito mais clara ainda com as exposições actuais. Obtivemos 12 odds ratios todos (fortemente) significativos na equação, explicando o facto de se sentir com má saúde pelas exposições a riscos (sem incluir, obviamente, as variáveis de controlo). O nervosismo (21 odds ratios significativos), as perturbações de sono (14), as preocupações (14), a fadiga (13), a ingestão de soníferos (12), as diferentes dores péri-articulares (de 8 a 14 odds ratios significativos), todas estas infra-patologias estão fortemente correlacionadas com declarações sobre as exposições.

\subsection{Os modelos estáticos completos não refutam a abordagem naïve}

11 A passagem aos modelos estáticos completos (controlando o efeito das características pessoais, dos comportamentos de risco, assim como de outras patologias e infrapatologias dos assalariados) conduz a muito poucas mudanças nos resultados. Perto de $80 \%$ das exposições que tinham um efeito significativo sobre a saúde nos modelos naïfs conservam um efeito significativo nos modelos estáticos completos (quadro 1). Os odd ratios que perdem a sua significância são aqueles cuja probabilidade de diferir da unidade estava perto de $5 \%$ e que passam acima desse nível nos modelos estáticos completos. 
Quadro 1 : Comparação do número de exposições actuais tendo um efeito significativo num modelo (linha) em relação a um modelo de referência (coluna)

\begin{tabular}{|c|c|c|c|c|c|c|}
\hline & \multicolumn{3}{|c|}{$\begin{array}{c}\text { Número de coeficientes } \\
\text { significativos nos modelos... }\end{array}$} & \multicolumn{3}{c|}{$\begin{array}{c}\text { Parte de coeficientes } \\
\text { significativos nos modelos... }\end{array}$} \\
\hline & Nalfs & $\begin{array}{c}\text { Comple- } \\
\text { tos }\end{array}$ & $\begin{array}{c}\text { Retro- } \\
\text { spectivos }\end{array}$ & Nalfs & $\begin{array}{c}\text { Comple- } \\
\text { tos }\end{array}$ & $\begin{array}{c}\text { Retro- } \\
\text { spectivos }\end{array}$ \\
\hline Nalfs & 269 & 215 & 215 & $100 \%$ & $80 \%$ & $80 \%$ \\
\hline Completos & & 234 & 212 & $92 \%$ & $100 \%$ & $91 \%$ \\
\hline Retrospectivos & & & 295 & $73 \%$ & $72 \%$ & $100 \%$ \\
\hline $\begin{array}{l}\text { Quadro 1: Comparação do número de exposiç̃es actuais tendo um efeito significativo num modelo } \\
\text { (linha) em relação a um modelo de referência (coluna) }\end{array}$
\end{tabular}

Fonte : ESTEV 1995

Nota de Leitura : $92 \%$ dos efeitos significativos nos modelos completos são significativos nos modelos naïfs - ou seja : entre os 234 efeitos significativos nos modelos completos, contamos 215 que são também significativos nos modelos naîfs.

De modo recíproco, quase todos (i.e., $92 \%$ ) os efeitos significativos relativos às exposições profissionais nos modelos completos, permanecem significativos nos modelos naïfs. A convenção de comparabilidade dessas duas famílias de modelos é aqui simplesmente a medida comparada do número de odds ratios significativos a $5 \%$. Um número quase equivalente de efeitos é portanto revelado pelos modelos naïfs e os modelos completos, com uma ligeira tendência dos primeiros para sobrestimar os efeitos (269 coeficientes significativos para o primeiro tipo de modelos, contra 234 para o segundo), ainda que os valores dos odds ratios das duas modelizações sejam muito próximos (gráfico 1). 


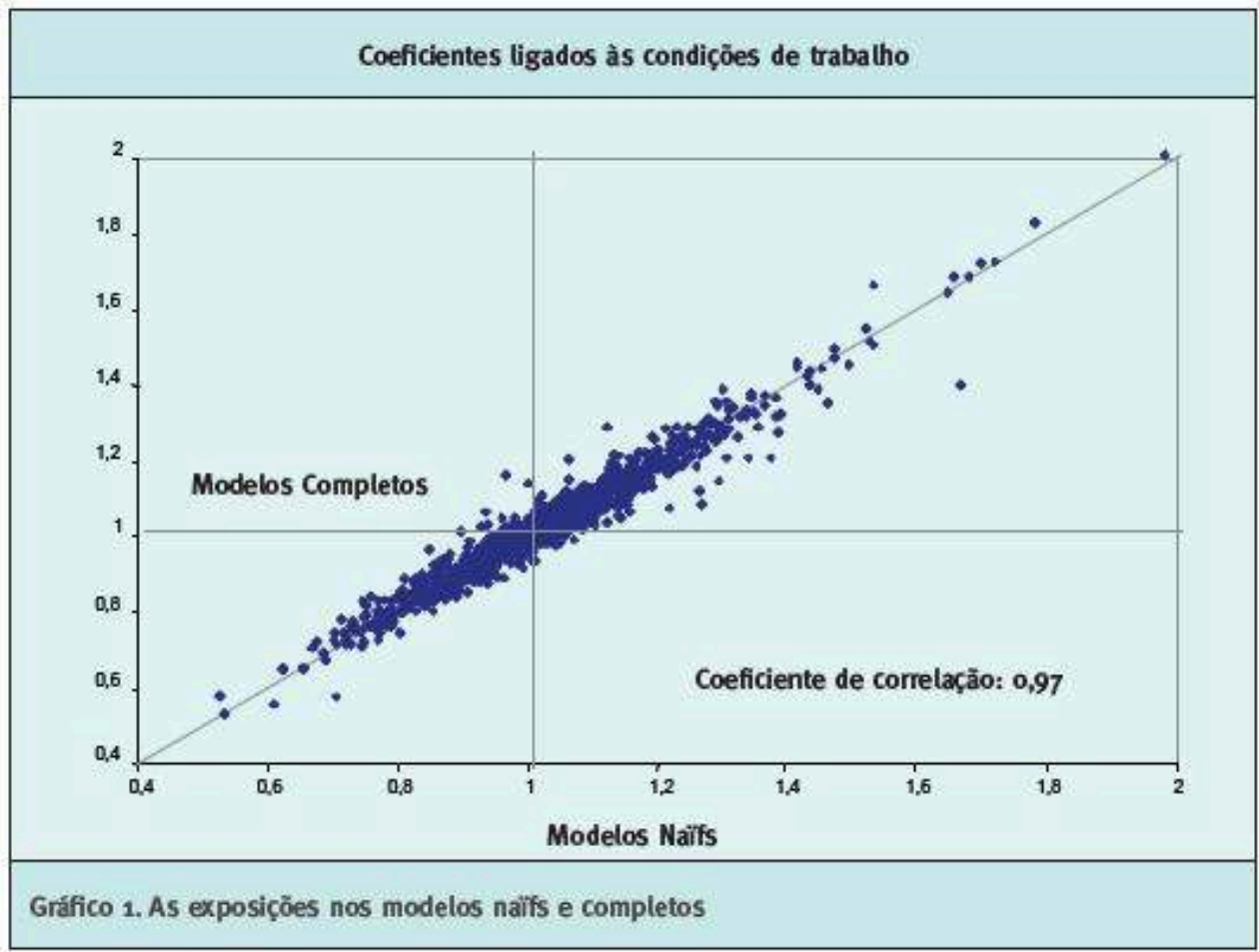

13 As esferas profissional e pessoal parecem assim agir de modo suficientemente independente sobre o estado de saúde das pessoas, para que a omissão de características pessoais e comportamentos de risco não enviese muito fortemente a medida das relações entre as exposições profissionais e o estado de saúde dos assalariados. De facto, dos modelos completos aos modelos naïfs, os valores assumidos pelos coeficientes significativos variam apenas um pouco: a maioria dos odds ratios significativos nos modelos naïfs vêem o seu valor aproximar-se ligeiramente de 1 no modelo estático completo correspondente, mantendo-se significativamente diferente da unidade.

14 A omissão das variáveis relacionadas com a vida pessoal dos inquiridos leva, deste modo, a sobrestimar ligeiramente o impacto das condições de trabalho actuais sobre a saúde, mas sem por isso enviesar a interpretação.

\subsection{A abordagem retrospectiva tem um melhor desempenho do que a abordagem naïve, mas não a invalida}

O inquérito ESTEV permite operacionalizar os modelos retrospectivos através da introdução de variáveis descrevendo o histórico das exposições passadas (por outras palavras, o facto de ser ou ter sido exposto a certos riscos identificados, assim como a duração eventual da exposição). Para os riscos físicos e os horários atípicos (mas não para os riscos organizacionais), o questionário ESTEV inclui uma interrogação dupla sobre a actualidade, por um lado, e a duração da exposição, por outro. Podemos assim decompor a exposição eventual a um risco em quatro componentes :

- uma exposição actual curta (i.e., exposição no momento do inquérito há menos de 10 anos)

- uma exposição actual longa (i.e., no momento do inquérito há mais de 10 anos) 
- uma exposição passada curta (i.e., anterior ao inquérito e durante menos de 10 anos)

- uma exposição passada longa (i.e., anterior ao inquérito e durante mais de 10 anos) naïfs e completos), quatro odds ratios são agora calculados para as exposições que o permitem. Na medida em que as declarações dos inquéritos são fiáveis (o que não é inteiramente o caso - Molinié \& Laville, 2000 ; Molinié, 2003), estes modelos permitem ter em conta o impacto dos antecedentes profissionais dos inquiridos.

Os efeitos dos constrangimentos organizacionais, para os quais o questionário ESTEV não prevê uma interrogação retrospectiva, permanecem muito próximos daqueles realçados pelos modelos naïfs (Coutrot \& Wolff, 2005). Para as outras exposições estimamos quatro coeficientes, o que complica a comparação com os modelos naïfs. Se começarmos por examinar o efeito das exposições actuais (curta ou longa), tendo presente como critério um nível de significância de 0,05 de pelo menos um destes dois coeficientes, os modelos retrospectivos fazem aparecer mais efeitos significativos do que os modelos naïfs e estáticos completos. Mas os modelos naïfs não se revelam massivamente infirmados. Num caso em cada cinco, um efeito significativo nos modelos naïfs não o é mais nos modelos retrospectivos (quadro 1), o que significa a contrario que $80 \%$ dos efeitos constatados na abordagem naïve são confirmados pela abordagem retrospectiva.

Quadro 2 : 0 impacto das exposições [4] sobre a saúde nos modelos retrospectivos

\begin{tabular}{|c|c|c|c|c|}
\hline & \multicolumn{4}{|c|}{ Námero de aparecimentos enquanto factor de ... } \\
\hline & Desgaste & Prevençăo & $\begin{array}{l}\text { Seleccăo } \\
\text { ex-ante }\end{array}$ & $\begin{array}{l}\text { Selecçäo } \\
\text { ex-post }\end{array}$ \\
\hline \multicolumn{5}{|c|}{ Exposiçoes mais do tipo "desgaste" } \\
\hline Ruido & ${ }_{11}$ & & & \\
\hline calor & 4 & 1 & & \\
\hline Esforcos sobre máquinas & 8 & 1 & & 1 \\
\hline Carregamento de cargas pesadas & 6 & $=$ & & \\
\hline Postura penosa & ${ }^{27}$ & 3 & & \\
\hline Poeiras & 8 & 1 & & \\
\hline Remuneraçăo por rendimento & 5 & 2 & & \\
\hline Semana de mais de $48 \mathrm{~h}$ & 6 & 1 & & \\
\hline $\begin{array}{l}\text { Trabalho repetitivo sob } \\
\text { constrangimento de tempo }\end{array}$ & 10 & ${ }^{3}$ & & \\
\hline \multicolumn{5}{|c|}{ Exposiç̄es dificilmente dassificíveis } \\
\hline Agentes infecclosos & 7 & 6 & & \\
\hline Deslocaçōes a pé frequentes ou rapidas & 6 & 5 & & \\
\hline Em attemância & & 3 & 1 & \\
\hline Firo & 3 & 4 & & \\
\hline Horários tardilos & 3 & 4 & & \\
\hline Intempéries & 2 & 3 & & \\
\hline Produtos químicos & 3 & 2 & & 1 \\
\hline Remuneraçăo por objectivo & 6 & 5 & 1 & \\
\hline Vibraç̄oes & 5 & 4 & & \\
\hline \multirow[t]{2}{*}{ Total } & 110 & 50 & 2 & 2 \\
\hline & $67,1 \%$ & $30,5 \%$ & $1,2 \%$ & $1,2 \%$ \\
\hline
\end{tabular}

Fonte : ESTEV 1995

Nota de leitura : nos 29 modelos utilizados, a "postura penosa" aparece 17 vezes como factor de desgaste e 3 vezes como factor de prevenção.

O exame dos resultados dados pelos modelos retrospectivos permite distinguir quatro tipos de efeitos das exposições actuais sobre a saúde (quadro 2), que escolhemos 
baptizar com nomes ilustrativos ("prevenção", "desgaste”, "selecção"), para facilitar a leitura mas sem pretender identificar os mecanismos etiológicos exactos.

- Os efeitos ditos de "prevenção" são aqueles para os quais os odds ratios significativos correspondendo às exposições actuais curta e longa, são inferiores à unidade (i.e., os dois odds ratios são significativos e inferiores a 1, ou então, só um dos dois o é e o outro é nãosignificativo a um nível de significância de 0,05$)$; falamos em "prevenção" porque a exposição parece proteger contra a patologia a curto e/ou médio prazo.

- Os efeitos ditos de "desgaste" são aqueles para os quais os odds ratios significativos são superiores à unidade : a exposição parece degradar a saúde, a curto e/ou médio prazo.

- Os efeitos ditos de "selecção" ex-ante são aqueles para os quais o odd ratio de exposição actual curta é (significativamente) inferior à unidade e o da exposição actual longa é (significativamente) superior à unidade : dito de outra forma, os assalariados submetidos há pouco tempo à exposição estão em melhor saúde do que os outros, mas aqueles que a ela estão submetidos há mais tempo têm uma saúde degradada ; essa situação poderá reflectir uma selecção sobre a saúde dos candidatos que se opera à "entrada" dos postos de trabalho, no momento do recrutamento.

- Os efeitos ditos de "selecção" ex-post são aqueles para os quais se observa a configuração inversa: a interpretação etiológica seria que os assalariados recentemente expostos sentissem rapidamente os efeitos nefastos da exposição, os assalariados que conseguem ter o domínio destes efeitos permanecem de modo durável nestes postos.

19 Sobre as 522 relações exposição-saúde analisadas (18 exposições por 29 (infra)patologias, identificamos, na realidade, quatro casos de efeito de "selecção". Para dois terços dos casos, os odds ratios significativos reenviam aos efeitos de "desgaste", mas num terço dos casos, os coeficientes evocam efeitos não unívocos, de "desgaste" para certas (infra)patologias e de "prevenção" para outras. (Lembremos que não procuramos aqui avaliar a pertinência epidemiológica das relações identificadas, mas as suas qualidades estatísticas).

Para comparar as performances das abordagens naïve e retrospectiva, reteremos a convenção seguinte : um coeficiente estimado como significativo num modelo naïf é coerente com a abordagem retrospectiva quando o seu sinal (relativamente à unidade) corresponde ao sinal do coeficiente (significativo) da mesma exposição no modelo retrospectivo, que é incoerente se não corresponde e, se corresponder, é compatível. Através desta convenção de comparabilidade, as duas abordagens parecem bastante coerentes (quadro 3) : a maior parte dos efeitos estimados como significativos pelos modelos naïf continuam a sê-lo nos modelos retrospectivos (sobre os 113 coeficientes significativos nos modelos naïfs, apenas 21 correspondem a efeitos não-significativos nos modelos retrospectivos) e nenhuma incoerência surgiu entre as duas abordagens. 
Quadro 3 : Coerência dos modelos naïfs e retrospectivos

\begin{tabular}{|c|c|c|c|c|c|c|c|}
\hline & & & \multicolumn{4}{|c|}{ Modelos Retrospectivos } & \multirow{3}{*}{ Total } \\
\hline & & & \multicolumn{3}{|c|}{ Significativos } & \multirow{2}{*}{$\begin{array}{l}\text { Não } \\
\text { Significa- } \\
\text { tivos }\end{array}$} & \\
\hline & & & $\begin{array}{l}\text { "Des- } \\
\text { gaste» }\end{array}$ & $\begin{array}{c}\text { «Preven- } \\
\text { ção» }\end{array}$ & $\begin{array}{c}\text { «Se- } \\
\text { lecção* }\end{array}$ & & \\
\hline \multirow{4}{*}{$\begin{array}{l}\text { 다 } \\
\text { है } \\
\text { ㅇ } \\
\frac{0}{2} \\
\sum\end{array}$} & \multirow{3}{*}{$\begin{array}{l}\text { Significa- } \\
\text { tivos }\end{array}$} & Coerentes & 68 & 23 & & & 91 \\
\hline & & $\begin{array}{l}\text { Incoer- } \\
\text { entes }\end{array}$ & & & & & o \\
\hline & & $\begin{array}{l}\text { Com- } \\
\text { pativeis }\end{array}$ & & 1 & & 21 & 22 \\
\hline & \multicolumn{2}{|c|}{ Não Significativos } & 42 & 26 & 4 & 337 & 409 \\
\hline \multicolumn{3}{|c|}{ Total } & 110 & 50 & 4 & 358 & 522 \\
\hline
\end{tabular}

Fonte : ESTEV 1995

Nota : este quadro apenas se aplica às 18 exposições para as quais o questionário ESTEV de 1995 prevê uma interrogação retrospectiva : 18 exposições em 29 (infra)patologias, ou seja 522 efeitos nos modelos naïs como nos modelos retrospectivos.

Nota de leitura : entre os 113 coeficientes significativos que contam os modelos naïfs, 91 são coerentes com os modelos retrospectivos (68 são de efeitos de desgaste, os outros 23 de prevenção) e os outros 22 são compatíveis. Nenhum entre eles faz aparecer a incoerência.

21 De modo inverso, muitos dos efeitos julgados significativos nos modelos retrospectivos não o eram nos modelos naïfs. É mais o caso de efeitos ditos "de prevenção", onde alguns parecem difíceis de interpretar de um ponto de vista epidemiológico. Os modelos naïs poderiam talvez parecer mais robustos do que os modelos retrospectivos, se não realizássemos uma análise epidemiológica sistemática das relações identificadas.

No total, a grande maioria (mais de 8 em 10) das relações significativas nos modelos naïfs continuam a sê-lo nos modelos retrospectivos, o que apoia a ideia de pertinência das relações reveladas pelos modelos naïfs : estes resultados não são infirmados pelas abordagens mais rigorosas. Por outro lado, os modelos retrospectivos parecem claramente ter um melhor desempenho no plano estatístico, porque perto de um terço das relações significativas que eles permitem identificar não apareceram nos modelos naïfs.

Podemos proceder da mesma forma para examinar os efeitos das exposições passadas sobre a saúde (quadro 4). As exposições passadas têm menos frequentemente efeitos significativos que as exposições actuais, mas os efeitos existentes vão mais frequentemente no mesmo sentido. Perto de metade das exposições tendo efeitos de desgaste, quando estão ainda presentes, vêem esse efeito confirmado quando examinamos as exposições passadas. Além disso, 75 odds ratios não significativos, no que concerne às exposições actuais, tornam-se significativos quando temos em conta as exposições passadas: a dimensão retrospectiva permite de facto enriquecer notavelmente a análise. 
Quadro 4 : Comparação dos efeitos das exposições actuais e passadas sobre a saúde nos modelos retrospectivos

\begin{tabular}{|c|c|c|c|c|c|c|c|}
\hline & & \multicolumn{5}{|c|}{ Exposiçōes actuais } & \multirow{2}{*}{ Total } \\
\hline & & Desgaste & Prevenção & $\begin{array}{l}\text { Selecção } \\
\text { ex-ante }\end{array}$ & $\begin{array}{l}\text { Selecção } \\
\text { ex-post }\end{array}$ & $\begin{array}{l}\text { Não Sig- } \\
\text { nificatívos }\end{array}$ & \\
\hline \multirow{5}{*}{ 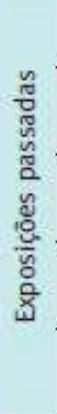 } & Desgaste & 47 & 2 & & 1 & 48 & 98 \\
\hline & Prevenção & 2 & 4 & & & 26 & 32 \\
\hline & $\begin{array}{l}\text { Selecção } \\
\text { ex-ante }\end{array}$ & & 1 & & & 1 & 2 \\
\hline & $\begin{array}{l}\text { Seleç̧ão } \\
\text { ex-post }\end{array}$ & & 1 & & & & 1 \\
\hline & $\begin{array}{l}\text { Não Sig- } \\
\text { nificativos }\end{array}$ & 61 & 42 & 2 & 1 & 283 & 389 \\
\hline & Total & 110 & 50 & 2 & 2 & 358 & 522 \\
\hline
\end{tabular}

Fonte : ESTEV 1995

Nota : este quadro apenas se aplica às 18 exposições para as quais o questionário ESTEV de 1995 prevê uma interrogação retrospectiva : 18 exposições em 29 (infra)patologias, ou seja 522 exposições

Nota de leitura : Entre os 110 efeitos de desgaste que foram identificados entre as exposições actuais, 47 são também de efeitos de desgaste quando consideramos as exposições passadas, 2 são efeitos de prevenção e 61 não aparecem como efeitos significativos.

\section{Considerar a dimensão longitudinal}

Graças à dupla interrogação em 1990 e 1995, os modelos longitudinais permitem modelizar não só a prevalência mas a incidência das (infra)patologias, num horizonte de cinco anos, e de pôr em evidência, de forma teoricamente mais precisa, as relações de causalidade entre exposições e a saúde. A consideração dos efeitos temporais já não é limitada às únicas exposições para as quais dispomos de informação puramente retrospectiva.

A priori, são possíveis duas abordagens longitudinais : modelizar a incidência de uma (infra)patologia em 1995 em função das características individuais e profissionais em 1990 (modelos longitudinais em nível), ou em função da evolução das exposições entre 1990 e 1995 (modelos longitudinais em variações).

\subsection{Longitudinal : sim, mas qual?}

O modelo longitudinal em nível é robusto no seguinte sentido : se existe uma correlação sistemática entre a exposição em 1990 e o aparecimento da patologia entre 1990 e 1995, é reduzido o risco de engano, quando se conclui uma relação de causalidade. Por outro lado, é enviesada, porque subestima estruturalmente a importância das relações. Pior ainda, pode levar a contra-senso. 
Consideremos a hipótese (evidentemente limite) em que uma patologia está intrinsecamente associada a uma exposição mas completamente reversível. Por exemplo, logo que o sujeito chega a um posto de trabalho exposto a fortes constrangimentos temporais, tem insónias ; a partir do momento em que o deixa, volta a um sono normal. Suponhamos, por outro lado, que a metade dos assalariados deixa de estar exposto entre 1990 e 1995, e que a outra metade começa a sê-lo: por outras palavras, opera-se um cruzamento completo entre expostos e não-expostos. Com a hipótese evocada, os expostos em 1995 dormiriam bem em 1990, e os expostos em 1990 dormem bem em 1995. Aqueles que passam a sofrer de insónias entre as duas datas são aqueles que não eram expostos em 1990 e que começaram a sê-lo em 1995.

o modelo longitudinal em nível (ou "standard") vai mostrar uma correlação perfeita entre a incidência da insónia e a ausência de exposição em 1990 : ou seja, a ausência de constrangimentos temporais parece provocar as insónias. Tratarse-á, evidentemente, de um perfeito artefacto estatístico e de um absurdo epidemiológico. O modelo longitudinal em variações permite evitar isso : com efeito, o sentido da causalidade é então restabelecido, e a correlação entre a incidência da patologia e o início da exposição é perfeita.

Podemos, sem dúvida, considerar que, quanto mais rápida é a associação entre exposição e patologia, mais a persistência da patologia depois do fim a exposição é breve, e mais o modelo standard se arrisca a subestimar os efeitos da exposição sobre a saúde, podendo mesmo, em casos extremos, sugerir associações francamente fantasistas. Certamente que o epidemiologista terá o cuidado de não as reter e as comentar, já que elas não terão nenhuma plausibilidade teórica, mas ele terá tendência a evocar uma aberração estatística, o que resulta, na realidade, de uma má escritura do modelo.

30 Por outro lado, o modelo em variações sofre de um outro tipo de limitação. Falar de causalidade supõe que a variação da exposição seja anterior à mudança do estado de saúde. Ora, não conhecemos a data de uma nem de outra: supõe-se que a saúde de certos indivíduos observados piore entre 1990 e 1995 antes de a sua exposição começar. A sua patologia não pode portanto ser atribuída à exposição. Contudo, a presença destes indivíduos na amostra contribuirá de modo enganador para a associação estatística entre o aparecimento da exposição e o da patologia. Tratar-se-á portanto de "observações parasitas", que podem então levar a passar além do nível de significância estatística para uma correlação, que não o passaria se as pudermos eliminar : de onde um risco de sobrestimação das relações entre exposição e patologia. Este risco não pode ser negligenciado na altura dos comentários respeitantes aos resultados dos modelos, cuja qualidade das estimativas deve ser avaliada (por exemplo, comparando os modelos calculados em diferentes sub-populações : homens e mulheres, profissões, etc.).

31 Um último problema metodológico importante diz respeito à precisão das informações sobre as exposições passadas e sobre a sua evolução entre as duas datas do inquérito. A.F. Molinié (2003) evidenciou as discordâncias entre as respostas de certos assalariados nas duas vagas (1990 e 1995) do ESTEV : assim, segundo as exposições, entre 10 e $35 \%$ das pessoas assinalando em 1990 ter estado expostas no passado, declaram em 1995 nunca ter estado expostas a essa exposição. Do mesmo modo, entre 5 e $15 \%$ dos assalariados que assinalam uma exposição actual em 1990, declaram em 1995 nunca a ter conhecido. Não se trata unicamente de erros de medida, mas também de reinterpretações : uma mudança de contexto pode levar o assalariado a mudar a sua 
avaliação de uma exposição passada - por exemplo, ocultando-a, porque ela parece muito secundária em relação aos novos problemas. 0 importante aqui é que Molinié (2003) mostra que estas discordâncias não estão ligadas ao estado de saúde das pessoas nem à sua evolução: assim, o ruído que introduzem nos modelos fragilizará a sua precisão estatística, mas não produzirá, provavelmente, um enviesamento sistemático. Retivemos, então, a modelização longitudinal em variações como referência, que comparamos sucessivamente com os modelos naïfs e longitudinais em nível.

\subsection{Os efeitos "de desgaste" são os mais numerosos na modelização longitudinal em variações}

Comparamos o impacto da exposição "permanente" (em 1990 e em 1995) ao do aparecimento de exposição entre 1990 e 1995, no seio do modelo explicativo da incidência de uma (infra)patologia entre 1990 e 1995. Como para os modelos retrospectivos, podemos distinguir na abordagem longitudinal quatro tipos de efeitos segundo o perfil temporal dos efeitos observados (quadro 5). Os efeitos de "prevenção" dizem respeito às exposições em que a incidência (unicamente em 1995) como a permanência (em 1990 e em 1995) agem no mesmo sentido favorável. Por exemplo, ter um trabalho "que permite aprender coisas novas", necessitando de "gestos muito precisos, muito minuciosos", parece proteger os assalariados dos PME, a curto ou a médio prazo (Gollac \& Volkoff, 2007). Pelo contrário, posturas penosas, sentadas, o carregamento de cargas pesadas, o facto de dever sempre despachar-se ou de ter horas de refeição irregulares, têm um papel enquanto factores de desgaste : o aparecimento de uma exposição em 1995, assim como a sua permanência entre 1990 e 1995, contribui para o aumento dos riscos de incidência de uma doença ósteo-articular ou muscular entre 1990 e 1995. 
Quadro 5 : Modelização longitudinal dinâmica

\begin{tabular}{|c|c|c|c|c|}
\hline $\begin{array}{l}\text { Número de aparecimentos de cada } \\
\text { exposiçảo com os efeitos de... }\end{array}$ & $\begin{array}{l}\text { Prevenção } \\
\text { (a) }\end{array}$ & $\begin{array}{l}\text { Desgaste } \\
\text { (b) }\end{array}$ & $\begin{array}{l}\text { Selecçâo } \\
\text { ex-ante } \\
\text { (c) }\end{array}$ & $\begin{array}{l}\text { Selecçāo } \\
\text { ex-post } \\
\text { (d) }\end{array}$ \\
\hline \multicolumn{5}{|c|}{ Exposiçōes mais preventivas } \\
\hline $\begin{array}{l}\text { Meios para fazer um trabalho de boa } \\
\text { qualidade }\end{array}$ & 9 & 0 & o & 1 \\
\hline Deslocações a pé frequentes ou rápidas & 5 & 1 & 1 & 1 \\
\hline Gestos minuciosos & 5 & 3 & 0 & 1 \\
\hline Exposição a produtos químicos & 5 & 2 & 1 & 1 \\
\hline Trabalho instrutivo & 5 & 1 & 1 & o \\
\hline Trabalho variado & 5 & 2 & 0 & 1 \\
\hline Escolha do modo de proceder & 4 & 2 & o & o \\
\hline Trabalho ao fim-de-semana & 4 & 2 & 1 & 1 \\
\hline Frio & 2 & 1 & o & o \\
\hline
\end{tabular}

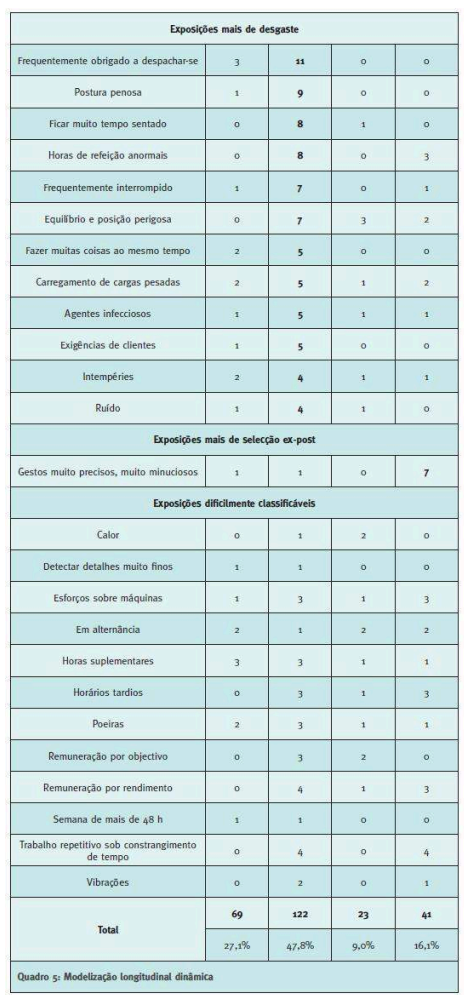

Fonte : ESTEV 1990 et 1995

Nota de leitura : ter os "meios suficientes para fazer um trabalho de qualidade" tem um papel de "prevenção" (com um coeficiente num nível de significância de 0,05) em 9 modelos dinâmicos sobre 29. 
nos modelos retrospectivos, os efeitos ditos "de desgaste" são os mais frequentes e são muitas vezes associados a exposições a riscos físicos (postura penosa ou sentada, "manter-se em equilíbrio em situações perigosas", carregamento de cargas pesadas...). Têm um papel semelhante um certo número de penosidades ligadas aos horários atípicos (horas de refeição "irregulares ou anormais"”), aos constrangimentos de ritmo ("ser frequentemente obrigado a despachar-se") ou à carga mental ("ser frequentemente interrompido no seu trabalho", "dever suportar as exigências do público, dos clientes").

As variáveis mobilizando os efeitos ditos "de prevenção" são um pouco menos frequentes, mas têm um papel claro : eles reenviam sobretudo para características da organização do trabalho, essencialmente sob o ângulo das oportunidades que oferecem ao assalariado para desenvolver as suas capacidades (ter os "meios para fazer um trabalho de boa qualidade", trabalho que "permite aprender coisas", "trabalho variado", poder "escolher (ele próprio) o modo de proceder"). A exposição ao frio ou as deslocações a pé frequentes ou rápidas aparecem também mais como "preventivas" e as formas de renumeração (por rendimento ou por objectivo) mais como factores "de desgaste".

Os efeitos ditos de "selecção ex-ante" e "ex-post" aparecem aqui também de modo esporádico.

\subsection{Os modelos naïfs não são infirmados pela abordagem longitudinal}

Entre a modelização naïve e a modelização longitudinal em variação, a comparação directa do número de odds ratios significativos torna-se pouco relevante, porque o modelo longitudinal comporta, por construção, quatro vezes mais odd ratios que o modelo naïf. É preciso, então, fazer-se uma nova convenção de comparabilidade. Seja uma exposição associada positivamente a uma patologia no modelo naiff (odd ratio $>1$ num nível de significância de 0,05 no modelo naïf ). Diremos que a modelização longitudinal em variações é "coerente" com a abordagem naïve no que diz respeito a essa patologia e exposição se (simultaneamente) :

- o odd ratio associado ao aparecimento dessa exposição entre 1990 e 1995, sendo significativo, é superior a 1 ;

- o odd ratio associado à mantimento dessa exposição, sendo significativo, é superior a 1 ;

- o odd ratio associado ao desaparecimento dessa exposição, sendo significativo, é inferior a 1 ;

- e um dos três odds ratios precedentes é significativo. Pelo contrário, diremos que os dois modelos são incoerentes se o aparecimento ou a mantimento da exposição reduzem significativamente a probabilidade de patologia, ou se o desaparecimento da exposição aumenta significativamente a probabilidade de patologia. Enfim, os dois modelos serão considerados como compatíveis se não são nem coerentes, nem incoerentes. Este critério é modificado de modo adequado no caso em que o odd ratio do modelo naif é inferior à unidade. 0 quadro 6 apresenta uma síntese desta comparação sistemática entre os modelos naïfs e longitudinais em variações. Em 269 relações significativas nos modelos naïfs, 139 (51\%) são coerentes com a modelização em variações, 112 (42\%) são compatíveis e unicamente 18 (7 \%) são incoerentes. Dito de outro modo, metade das relações identificadas 
nos modelos naïfs é confirmada pelos modelos em variações, uma outra (pequena) metade não é confirmada nem infirmada e uma pequena minoria parece infirmada.

Quadro 6: Comparação dos modelos naïfs e longitudinais em variações

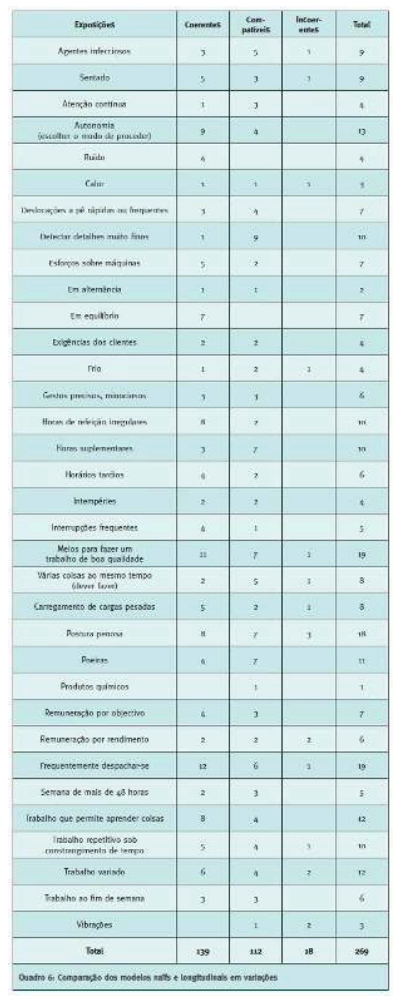

Fonte : ESTEV 1990 et 1995

Ajuda na leitura : sobre 9 coeficientes significativos (num nível de significância de 0,05) dizendo respeito aos agentes infecciosos nos modelos naïfs, 3 são coerentes com os correspondentes modelos longitudinais em variações, 5 são compatíveis e 1 é incoerente. em variações eles-mesmos. Tomemos o exemplo das posturas penosas, para as quais três incoerências são observadas. Em dois destes casos, é o modelo em variações que aparece incoerente, já que tanto o aparecimento como o desaparecimento da exposição (assim como a sua permanência) aumentam significativamente a probabilidade da infra-patologia (isto é, as "preocupações que impedem de dormir", declaradas pelo assalariado, e as perturbações de sono diagnosticados pelo médico). De modo semelhante, o modelo explicando os PME do joelho pelo facto de ser "frequentemente obrigado a despachar-se" revela um odd ratio de 1,4 para o aparecimento da exposição, mas também de 1,2 para o seu desaparecimento, os dois tendo um nível de significância de 0,05 : é então contraditório, segundo os critérios acima definidos, com o modelo naïf (odd ratio de 1,2) mas a contradição é de facto interna ao modelo em variações em si. No total, 13 das 18 incoerências resultam deste tipo de caso, o que reduz para 5 (apenas $2 \%)$ o número de relações exposição-patologia onde podemos realmente considerar que a modelização longitudinal em variações infirma os modelos naïfs.

relações exposição-patologia ditas "compatíveis" são aquelas onde o modelo naïf indica um efeito significativo da exposição sobre a patologia, mas onde nenhum odd ratio é significativo no modelo longitudinal em variações. $O$ modelo naïf sobrestimava, então, o impacto da exposição. Frequentemente, o odd ratio da variável "permanência 
da exposição nos dois inquéritos", ainda que não significativo, dá a mesma indicação que o odd ratio da exposição em 1995 no modelo naîf : em 80 \% dos casos os dois odd ratios são do mesmo lado da unidade.

Por fim, parece que ter em consideração unicamente os modelos naïfs não conduz a grandes contra-sensos : é apenas num número insignificante de casos que os modelos longitudinais em variações contradizem claramente o que sugerem os modelos naïfs. Por outro lado, os modelos naïfs fornecem frequentemente resultados um pouco "forçados", já que a sua significância desaparece, frequentemente, nos modelos longitudinais em variações.

\subsection{Uma tentativa de comparação global das diversas abordagens}

Construímos a título puramente indicativo e de forma exploratória um indicador de "performance global" dos modelos, atribuindo a cada relação exposição-patologia o valor 3 se o odd ratio associado tem um nível de significância de 0,$01 ; 2$ num nível de significância de 0,$05 ; 1$ num nível de significância de 0,1 e 0 se não é significativo num nível de significância de 0,1 e somando estes valores para o conjunto das exposições de cada modelo (cf. quadro 7, onde as (infra)patologias são classificadas em função do número de odd ratios significativos que obtêm nos modelos naïfs). Segundo esta nova convenção de comparabilidade, para uma dada patologia, quanto mais um modelo comporta odd ratios significativos, e quanto mais esta significância é forte, mais o modelo será considerado como performante para explicar a patologia. 
Quadro 7 : Performances relativas dos modelos segundo as patologias (performances calculadas unicamente sobre os odds ratios associados às exposições)

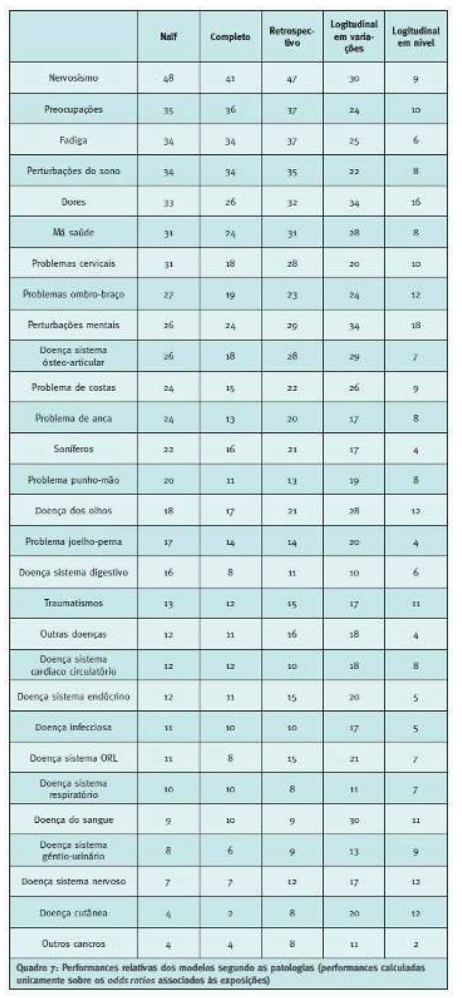

Fonte : ESTEV 1990 et 1995

Ajuda na leitura : no modelo naïf respeitante ao nervosismo, a soma ponderada das significâncias fazem 48 (significância : 3 para um coeficiente significativo a um nível de 0,$01 ; 2$ para um nível de 0,$05 ; 1$ para um nível de 0,1 ). Para os modelos naïfs, completos e longitudinais em nível, o indicador de performance é construído segundo esta fórmula de soma ponderada. Para os modelos retrospectivos (no caso em que dois coeficientes são dados para uma mesma característica exposição actual curta e actual longa) e os modelos em variações, a fórmula é mais elaborada, já que há mais que um coeficiente por característica do trabalho. Ema característica tem, então, notação 3 logo que, para uma característica dada, um desses coeficientes associados seja significativo a um nível de 0,01 ; ou 2 quando um dos coeficientes pelo menos é significativo a 0,05, etc....

Este quadro deixa várias indicações interessantes. Primeiro, a "performance" dos modelos naïfs aparece muito próxima da dos modelos longitudinais em variações para as infra-patologias (nervosismo, tomada de soníferos, perturbações de sono...) ou para as perturbações ou patologias ósteo-articulares, assim como para os traumatismos, ou mesmo as perturbações mentais. Estas perturbações da saúde são directamente ligadas a exposições actuais no trabalho, e o diagnóstico dos modelos naifs não é, em geral, infirmado pelos modelos mais sofisticados.

Pelo contrário, e isto era esperado, para as patologias que podem resultar sobretudo do efeito diferido das exposições (doenças do sistema respiratório, circulatório ou genital, doenças do sangue, cancros...), o poder explicativo dos modelos naïfs é muito fraco. Os modelos em variações, estes, são tanto performantes para estas doenças como para as infrapatologias ou os PME, o que confirma a sua maior pertinência para avaliar, na generalidade, os efeitos das condições de trabalho sobre a saúde.

Um outro resultado interessante diz respeito às performances relativas aos modelos longitudinais em nível e em variações. As duas famílias de modelos partilham esta propriedade de ser igualmente pertinentes para todos os tipos de patologias estudadas, 
diferentemente dos modelos naïfs. Por outro lado, os modelos longitudinais em nível aparecem mais pobres em poder explicativo, os indicadores de performance aqui calculados sendo sistematicamente inferiores aos dos modelos longitudinais em variações (quadros 7 e 8). Isto não é surpreendente, por um lado porque o modelo em variações faz intervir três odd ratios onde o modelo em nível comporta apenas um, e por outro lado, porque o modelo em nível tende, estruturalmente, a subavaliar o impacto das exposições sobre a saúde.

Quadro 8 : Testes dos pares concordantes e discordantes

\begin{tabular}{|c|c|c|c|c|}
\hline \multirow{2}{*}{} & \multicolumn{3}{|c|}{ Média de pares } \\
\cline { 2 - 5 } & Concordantes & Discordantes & Ligados \\
\cline { 2 - 5 } & Simples & $62,7 \%$ & $35,0 \%$ & $2,3 \%$ \\
\cline { 2 - 5 } & Completos & $70,0 \%$ & $28,5 \%$ & $1,5 \%$ \\
\cline { 2 - 5 } & Retrospectivos & $71,1 \%$ & $27,5 \%$ & $1,4 \%$ \\
\cline { 2 - 5 } & Standards & $65,3 \%$ & $32,3 \%$ & $2,5 \%$ \\
\cline { 2 - 5 } & Em variações & $70,1 \%$ & $28,1 \%$ & $1,8 \%$ \\
\hline \multirow{2}{*}{ Quadro 8: Testes dos pares concordantes e discordantes } & & \\
\hline
\end{tabular}

\section{Fonte : ESTEV 1990 et 1995}

Nota de leitura : Os modelos naifs obtêm em média $62,7 \%$ de pares concordantes, conta 70,1 \% para os modelos em variações. O número de pares concordantes é um indicador do poder explicativo do modelo.

Igualmente como esperado, o intervalo de performance entre modelos em variações e em nível, aparece sobretudo para as (infra)patologias mais reversíveis, que resultam mais de efeitos a curto prazo das exposições. Excepto o caso (raro) das doenças do sangue, a diferença entre a quarta e a quinta coluna do quadro 7 tende a reduzir-se claramente, na parte inferior do quadro. Dito ainda de outro modo, o modelo em nível é claramente menos performante em relação ao modelo em variações, essencialmente no caso das perturbações psíquicas, quer se trate de sofrimento psíquico sentido pelo próprio ou de perturbações mentais diagnosticadas e de perturbações péri-articulares, quer se trate de dores declaradas ou de PME diagnosticados. Trata-se de uma fracção cada vez mais importante das perturbações de saúde ligadas ao trabalho.

\section{Discussão}

O nosso estudo estatístico chegou a quatro tipos de resultados. Em primeiro lugar, a não consideração dos factores pessoais, dos comportamentos de risco e dos antecedentes médicos, enviesa pouco a avaliação das relações entre exposições e saúde, pelo menos na amostra aqui estudada. 
47 Em segundo lugar, a consideração do histórico das exposições aos riscos profissionais não invalida os ensinamentos provenientes de uma modelização "naïve", no que diz respeito às relações entre exposições profissionais e saúde, mas enriquece claramente a análise.

Em terceiro lugar, se - como poderíamos esperar - os modelos naïfs não são de nenhuma utilidade para dar conta dos efeitos diferidos das exposições profissionais sobre a saúde no que diz respeito às patologias graves (doenças do sistema respiratório, nervoso, génito-urinário, cancros...), as suas indicações não são desmentidas - e são mesmo frequentemente confirmadas - por uma abordagem longitudinal no que diz respeito às infra-patologias, as perturbações psíquicas e musculo-esqueléticas. Dito de outro modo, não parece abusivo interpretar, em termos de causalidade, as correlações observadas em cross-section entre certas exposições profissionais (aquelas ligadas, nomeadamente, à organização do trabalho) e certas (infra)patologias, embora mantendo a prudência necessária.

49 Em quarto lugar, quando comparamos as abordagens longitudinais, a modelização em nível parece menos performante em relação à modelização em variações, para o que diz respeito às (infra)patologias associadas ao stress (perturbações psíquicas ou músculoesqueléticas), já que ela leva a minorar o impacto das exposições sobre a saúde. A sua utilização exclusiva arrisca, então, ocultar as consequências a curto prazo das condições e da organização do trabalho. A explicação é provavelmente relacionada com a reversibilidade das perturbações em caso de desaparecimento da exposição, que pode tornar a modelização em nível pouco operatória.

50 A experiência estatística aqui realizada repousa sobre a grande riqueza da base de dados ESTEV (informações simultaneamente retrospectivas e longitudinais sobre as exposições e sobre as patologias, informações sobre a situação pessoal e os comportamentos de risco, questionário médico e auto-avaliação da saúde). No entanto, os resultados obtidos são dificilmente generalizáveis, provavelmente, em parte, pelo grande tamanho da amostra, que limita o risco de aleatoriedades estatísticas, frequentes nas pequenas amostras, onde as correlações, aparentemente significativas, entre exposições e estado de saúde desaparecem com a consideração dos "factores de confusão". Estes resultados exigiriam ser confirmados por estudos semelhantes sobre outros dados apresentando as mesmas vantagens (importante tamanho da amostra, informações retrospectivas e longitudinais), como por exemplo o inquérito Saúde e Itinerários Profissionais (Ministério do Trabalho, 2007) depois da sua segunda aplicação, prevista em 2010.

51 Por outro lado, a comparação das performances de diversos modelos repousa sobre critérios convencionais, que qualificamos aqui de "convenções de comparabilidade", e poderão, sem dúvida, ser modificados pela escolha de outras convenções. A comparação dos modelos explicativos das diversas patologias pode, quanto a si, ser afectada pelo facto que os efectivos que revelam certas patologias são muito mais fracos que os tocados por outras (infra)patologias. Mas o principal limite deste estudo relaciona-se com o facto das comparações entre modelizações repousarem sobre critérios puramente estatísticos, independentemente da plausibilidade epidemiológica das relações exposição-patologia estudadas. Ora, nós vimos que esta plausibilidade parece, em certos casos, muito fraca. As performances estatísticas das diferentes modelizações dependem, então, em parte, de relações pouco interpretáveis no plano epidemiológico, o que pode manchar a sua comparação com enviesamentos dificilmente controláveis. 
Não parece contudo que esses artefactos constituam uma parte suficiente das relações identificadas para invalidar os resultados aqui apresentados.

\section{BIBLIOGRAFIA}

Bertin, C. \& Derriennic, F. (2001). Sentiment de lassitude, âge et conditions de travail. In Travail, Santé, Vieillissement, Relations et évolutions, Actes du Colloque des 18 et 19 novembre 1999. Toulouse : Octarès.

Bué, J., Coutrot, T. \& Puech, I. (2005). Conditions de travail : les enseignements de vingt ans d'enquêtes. Toulouse : Octarès.

Coutrot, T. \& Wolff, L. (2005). L'impact des conditions de travail sur la santé : une expérience méthologique. Documents d'études de la Dares, 2005-097, http://www.travail.gouv.fr/etudesrecherche- statistiques/etudes-recherche/publications-dares/les-documentsetudes/2005097impact-conditions-travail-sur-sante-uneexperience-methologique-2591.html

Goldberg, M. (2003). L'épidémiologie sans peine. Paris : Frison Roche. Derriennic, F., Touranchet, A. \& Volkoff, S. (1996). Age, travail, santé ; Etudes sur les salariés de 37 à 52 ans, résultats de l'enquête ESTEV. Paris : Éditions de l'INSERM.

Derriennic, F., Monfort, C., Cassou, B. \& Touranchet, A. (2001). Douleurs lombaires : les principales tendances observées dans l'enquête ESTEV. In Travail, Santé, Vieillissement, Relations et évolutions, Actes du Colloque des 18 et 19 novembre 1999. Toulouse : Octarès.

Gollac, M. \& Volkoff, S. (2007). Les conditions de travail. Paris : La Découverte.

Ministère de l'emploi et de la solidarité (2001). STED 1993-1998 : enquête longitudinale auprès de salariés DATR des entreprises sous-traitantes d'EDF intervenant lors des arrêts de tranche des centrales nucléaires.

Ministère du travail (2007). L'enquête Santé et itinéraires professionnels (SIP). http:// www.travail-solidarite.gouv.fr/etudes-recherche- statistiques/statistiques/sante-au-travail/ enquetes/sip-5353.html, 2007

Molinié, A. F. \& Laville, A. (2000). Les surprises du longitudinal : les salariés répondent-ils n'importe quoi aux enquêtes portant sur leur travail actuel et passé ?. In B. Mélier \& Y. Quéinnec (org.), Communication et travail, XXXVè Congrès de la SELF. Toulouse : Octarès.

Molinié, A. F. (2003). Interroger les salariés sur leur passé professionnel : le sens des discordances. Revue d'Épidémiologie et de Santé Publique, 51, 589-605.

Vézina, M., Derriennic, F. \& Monfort, C. (2001). Tension au travail et atteintes à la santé mentale : l'éclairage de l'enquête ESTEV. In Travail, Santé, Vieillissement. Relations et évolutions, Actes du Colloque des 18 et 19 novembre 1999. Toulouse : Octarès. 


\section{NOTAS}

1. Para uma apresentação mais detalhada dos métodos e dos resultados, ver Coutrot \& Wolff, 2005.

2. Isto é, se a mesma exposição intervém significativamente em cada modelo mas em sentidos opostos.

3. Lembramos que se trata dos efeitos das exposições passadas para os indivíduos que já não são submetidos a essas exposições no momento do inquérito.

4. Neste quadro apenas aparecem as características que deram lugar a uma interrogação retrospectiva

\section{RESUMOS}

Este artigo visa realizar uma comparação sistemática das performances dos modelos epidemiológicos ditos "naïfs", explicando a prevalência das perturbações de saúde através das únicas características actuais do trabalho dos assalariados, com modelos mais rigorosos no plano teórico, incluindo um histórico de certas exposições profissionais anteriores (modelos estatísticos retrospectivos), ou estudando a incidência (em lugar da prevalência) das perturbações em função, seja da exposição à data inicial (modelos longitudinais standard), seja da evolução da exposição (modelos dinâmicos).

Mostra-se num primeiro momento quanto o impacto da consideração de factores individuais de confusão - o consumo de álcool e de tabaco, os modos de vida as pessoas ou o seu historial médico - é fraco : as correlações entre a saúde e as condições actuais de trabalho evidenciadas pelos modelos naïfs permanecem inalteradas. Isso não significa, certamente, que esses factores não tenham efeitos na saúde das pessoas - pelo contrário, a análise mostra os efeitos importantes de alguns desses factores - mas os efeitos dos factores profissionais e dos factores pessoais agem de maneira largamente independente.

De seguida serão examinados os méritos respectivos dos modelos standard e dinâmicos: os primeiros explicando a incidência de uma perturbação de saúde entre duas datas pela exposição à data inicial, os segundos usando como variáveis explicativas a evolução da exposição entre as duas datas. No que respeita às (infra)patologias ligadas ao stress, os modelos standard aparentam ser menos performantes, na medida em que subestimam claramente o impacto das exposições sobre as perturbações de saúde. A explicação está provavelmente relacionada com a maior reversibilidade das perturbações em caso de desaparecimento da exposição, fenómeno que o modelo standard confunde com uma correlação negativa entre a exposição e a perturbação.

Este artículo intenta realizar una comparación sistemática del desempeño de los modelos epidemiológicos llamados "ingenuos", modelos que explican la prevalencia de problemas de salud únicamente a partir de las características del trabajo de los asalariados, utilizando modelos más rigurosos desde un plano teórico, es decir que consideran el historial de exposiciones laborales (modelos estadísticos retrospectivos), o que estudian la incidencia (en lugar de la prevalencia) de los problemas ya sea en función de la exposición inicial (modelos longitudinales estandar), o en función de la evolución de la exposición (modelos dinámicos).

Mostramos, en un primer momento, cuan débil es el impacto de los factores individuales de confusión - el consumo de alcohol y de tabaco, los modos de vida de las personas o su historial 
médico: las correlaciones entre salud y condiciones de trabajo puestas en evidencia por los modelos “ingenuos" prácticamente no se ven modificadas. Esto no significa, por supuesto, que estos factores no tengan efectos sobre la salud de las personas por el contrario, el análisis muestra efectos importantes de algunos de esos factores pero que los efectos de los factores profesionales y personales intervienen de manera ampliamente independiente. Examinamos luego los méritos respectivos de los modelos “ estandar " y “ dinámico » : los primeros explican la incidencia de un problema de salud entre dos fechas por la exposición a la fecha inicial; los segundos toman como variables explicativas la evolución de la exposición entre ambas fechas. En lo que a (infra) patologías ligadas al stress se refiere, los modelos estandar son sub-eficientes en la medida que sub-estiman netamente el impacto de las exposiciones sobre los problemas de salud. La explicación tiene seguramente que ver con una mayor reversibilidad de los problemas en caso de desaparición de la exposición, fenómeno que el modelo estandar confunde con una correlación negativa entre la exposición y el problema.

Cet article vise à réaliser une comparaison systématique des performances des modèles épidémiologiques dits " naïfs", expliquant la prévalence des troubles de santé par les seules caractéristiques actuelles du travail des salariés, avec des modèles plus rigoureux au plan théorique, incluant un historique de certaines expositions professionnelles passées (modèles statiques rétrospectifs), ou étudiant l'incidence (au lieu de la prévalence) des troubles en fonction soit de l'exposition à la date initiale (modèles longitudinaux standards), soit de l'évolution de l'exposition (modèles dynamiques).

On montre dans un premier temps combien l'impact de la prise en compte des facteurs individuels de confusion - la consommation d'alcool et de tabac, les modes de vie des personnes ou leur historique médical - est faible : les corrélations entre la santé et les conditions actuelles de travail mises en évidence par les modèles naïfs n'en sont pratiquement pas modifiées. Cela ne signifie bien sûr pas que ces facteurs sont sans effets sur la santé des personnes - au contraire, l'analyse montre les effets importants de certains de ces facteurs - mais que les effets des facteurs professionnels et des facteurs personnels jouent de façon largement indépendante.

On examine ensuite les mérites respectifs des modèles “standards » et “dynamiques »: les premiers expliquant l'incidence d'un trouble de santé entre deux dates par l'exposition à la date initiale, les seconds prenant pour variables explicatives l'évolution de l'exposition entre les deux dates. Pour ce qui concerne les (infra)pathologies liées au stress, les modèles standard apparaissent sous-performants, dans la mesure où ils sous-estiment nettement l'impact des expositions sur les troubles de santé. L'explication tient probablement à la plus grande réversibilité des troubles en cas de disparition de l'exposition, phénomène que le modèle standard confond avec une corrélation négative entre l'exposition et le trouble.

This paper intends to present a systematic comparison between the performances of ìnaiveî epidemiological models, explaining the prevalence of health issues through existing characteristics of workers, and more rigorous models that include a history of some former professional exposures (retrospective statistical models), or models that study the incidence (instead of the prevalence) of the disorders, according to either the exposure from the initial date (longitudinal standard models), or the evolution of the exposure (dynamic models). We first show the weakness of considering the impact of individual misleading factors such as the consumption of alcohol and tobacco, living conditions or medical history. However, the correlations between health and employment characteristics highlighted by the naive models remain practically unmodified in the more thorough models. That does not mean that these factors have no effects on health. On the contrary, the analysis shows the importance of some of these effects. But the effects of the professional factors and of the personal ones act independently.

We then examine the respective merits of ìstandardî and ìdynamicî models. The first ones 
explain the incidence of health disorders by exposure between two dates with reference to the initial date. The second ones take the evolution of the exposure between the two dates as explanatory variables. Concerning the (infra)pathologies related to stress, the ìstandardî models appear under-efficient, insofar as they clearly underestimate the impact of exposures on health disorders. This lack of efficiency is probably due to the greater reversibility of disorders in the case of the disappearance of exposure, a phenomenon that the ìstandardî model confuses with a negative correlation between the exposure and the disorder.

\section{ÍNDICE}

Palabras claves: modelos estadísticos en epidemiología, salud y trabajo, datos estadísticos, impacto de los factores individuales de confusión

Keywords: statistical models in epidemiology, health and working conditions, individual factors of confusion

Palavras-chave: modelos estatísticos em epidemiologia, saúde e trabalho, dados estatísticos, impacto dos factores individuais de confusão

Mots-clés: modèles statistiques en épidémiologie, santé et travail, données statistiques, impact des facteurs individuels de confusion

\section{AUTORES}

\section{THOMAS COUTROT}

Direction de l'Animation de la Recherche, des Etudes et des Statistiques (DARES), 39-43, quai André Citroën - 75015, Paris

thomas.coutrot@dares.travail.gouv.fr

\section{LOUP WOLFF}

Direction de l'Animation de la Recherche, des Etudes et des Statistiques (DARES), 39-43, quai André Citroën - 75015, Paris

loup.wolff@dares.travail.gouv.fr 